\title{
MODELLING CUSTOMERS' IMPATIENCE WITH DISCOURAGED ARRIVAL AND RETENTION OF RENEGING
}

\author{
PALLABI MEDHI* \\ Department of Statistics, Gauhati University, Guwahati 781014, Assam, India
}

\begin{abstract}
The paper presents stochastic modelling of a single server, finite buffer Markovian queueing system with discouraged arrivals, baulking, reneging, and retention of reneged customers. The Markov process is used to derive the steady-state solution of the model. Closed-form expressions using probability generating functions (PGFs) are derived and presented for both classical and novel performance measures. In addition, a sensitivity analysis is carried out to study the effect of the system parameters on performance measures. A numerical problem is also presented to demonstrate the derived results and some design aspects.
\end{abstract}

Keywords: baulking, impatience, queuing, reneging, discouraged arrival, retention of reneging

\section{Introduction}

In modelling real-life problems involving congestion, queueing theory plays a vital role as this theory analyses 'idealised' models, which typically may not exactly exist in practice but can serve as approximations ranging from reasonable to excellent. In today's competitive world, a customer decides to join the queue only when a short wait is required. Customers get impatient with the prospect of waiting. Such impatience translates into two types of customer behaviour, viz. baulking and reneging. Recently, these concepts were incorporated on a two-server Markovian model by Bouchentouf and Messabihi [3], Som and Kumar [21]. Applications of queueing with impatience can be seen in traffic modelling, business and industries, IT sectors, health sectors, medical sciences, and so forth (cf. [19]). It is important to note that customers' impatience has a very negative impact on the queueing system under investigation. In many situations, the arrival rate of customers into the system depends on the system size instead of a constant rate. This is known as discouraged arrival and is one form of state dependence. In

*Email address: pmedhi@gauhati.ac.in

Received 25 March 2021, accepted 8 June 2021 
this type of system, arrivals get discouraged from joining the queue when more and more people are present. Essentially, this results in lowering the arrival rate as the number of customers in the system increases. Natvig [18] first introduced the concept of discouraged arrival in $M / M / 1$ queue. Customers with impatience and discouragement have an impact on the system performance of standard queuing systems. Queues with discouraged arrivals have applications in computers with batch job processing where job submissions are discouraged when the system is frequently used and arrivals are modelled as a Poisson process with a state-dependent arrival rate. As stated earlier, this discouragement impacts the arrival rate of the queuing system.

On the other hand, if a customer on their arrival finds the queuing system nonempty, they might decide not to join the queue. In queuing parlance, this is known as baulking. Haight [9] provides a rationale that might influence a person to baulk. It relates to the perception of the importance of being served which induces an opinion somewhere in between urgency so that a queue of a certain length will not be joined to indifference where a non-zero queue is also joined. Baulking can be classified into two types, viz. state-independent baulking (SIB) and state-dependent baulking (SDB). In the case of SIB, the baulking rate remains constant in all the states of the system. Reversely, if baulking rate depends on the state of the system, we refer to it as SDB. In the second type of baulking, the probability that a customer will baulk increases as the queue length is higher, and where baulking probability is a function of the state of the system [16]. We shall assume SDB throughout this paper.

Again, some arriving customers do not baulk and decide to join the queue. Among those who join, it is commonly observed that some get impatient with waiting and leave the system without completely receiving service. This is known as reneging. Depending upon the particular time, customers could renege. Reneging customers can be of two types, viz. reneging till the beginning of service (referred to as R_BOS), and reneging till the end of service (referred to as R_EOS). R_BOS can be seen in queuing systems where customers can renege only as long as it is in the queue. Once service begins, it cannot renege. On the other hand, a customer can renege not only while waiting in a queue but also while receiving service in the case of R_EOS. A common example of the first type is customers queuing at a beauty parlour. In the parlour, once any kind of service (haircut, facial, etc.) starts, the customer cannot renege. Common examples of the second type are processing or merchandising of perishable goods, hospital emergency room/O.T., handling critical patients, etc. A critical patient may expire at the O.T. table of the emergency ward of a hospital which would be an example of reneging while service is going on.

When this impatience increases and customers leave before being served, some remedial actions must be taken to retain customers as this loss implies loss both in terms of immediate revenues as well as reputation to a business manager. The current business environment cannot afford this. One such remedial action considered in the literature involves providing an incentive for the customer not to renege. These incentives induce a desire on the impatient customer to retain in the system. Such an impatient customer 
(due to reneging) may hence be convinced to stay in the service system by introducing certain retention policies. Such customers are termed as retained customers. Under this framework, if a customer gets impatient (due to reneging), (s)he may leave the queue with probability say $p$ and may remain in the queue for service with probability $q$ such that $p+q=1[3,12]$. This is the concept referred to as retention of reneging customers.

The modelling approach of a single server finite buffer Markovian queuing model incorporating the additional challenges of discouraged arrival and state-independent baulking is the basis of this paper. The importance of the queuing model stems from the fact that in the classical $M / M / 1$ model, it is assumed that the system can accommodate any number of units. In practice, this may seldom be the case. We have thus to consider the situation such that the system has limited waiting space and can hold a maximum number of $k$ units (including the one being served [17]. Though this model has been analysed, however, to the best of our knowledge, the said model with the added complexity of discouraged arrival, retention of reneged customers, and state-dependent baulking has not been dealt with in the literature. Also, the closed-form expressions of various performance measures are not available for the said model. Only a restricted version of the single and multi-server queuing model for just two servers assuming impatient customers is available $[15,4]$. This work, therefore, aims to fill this gap in the literature.

In this paper, we shall analyse a single server Markovian queuing system $M / M / 1 / k$ with discouraged arrivals, baulking, reneging, and retention of reneged customers. As for baulking, we assume that each customer has a state-dependent baulking probability. It will be assumed that if the customer on arrival observes the system to be in state $i$, the probability that he will baulk is $i / k, i=1,2, \ldots, k$. With this set-up, the finite buffer restriction can also be seen as the state from which customer baulks with probability $1(k / k)$. It may be noted that our formulation requires that baulking is possible only when the system is non-empty. There is no baulking from an empty system. The arrival and service rates are assumed as $\lambda$ and $\mu$, respectively. We also assume that if a customer finding every server busy arrive with arrival rate that depends on the number of customers present in the system at that time, i.e., if there are $n(n>1)$ customers in the system, the new customer enters the system with rate $\lambda /(n+1)$. As for reneging, each customer joining the system is assumed to have random patience time following exp $v$. It is also assumed that when a customer gets impatient (due to reneging), (s)he may leave the queue with some probability, say $p$, and may remain in the queue for service with probability $q=1-p$.

The subsequent sections of this paper are structured as follows. Section 2 contains a literature review. Section 3 and section 4 contain the derivation of steady-state probabilities and performance measures. A numerical example is discussed in section 5 . We perform sensitivity analysis in Section 6. Concluding statements are presented in Section 7. The appendix presented in Section 8 contains some derivations. 


\section{Review of literature}

Even though one can observe reneging and baulking in our day-to-day life, it is not very often that one can locate a paper analysing these features simultaneously in a queuing system with the additional restriction of discouraged arrivals and retention of reneging customers $[13,10]$. Even if these were analysed, closed-form expressions of important performance measures are not available $[1,12,14]$. One of the recent works on baulking and reneging was carried out by Saikia, Medhi, and Choudhury [20] where they analyse a multi-server Markovian queuing system under the assumption that customers may baulk as well as renege. A specific baulking rule where the baulking probability of the customer decreases as the state of the system goes up, i.e., reverse baulking as well as position-dependent reneging (PDR), a very relevant formulation from the practical point of view, are considered in this paper. They derive the generating function of the stationary system size distribution and also obtain the mean system size along with the other performance measures. But they do not consider the concept of discouraged arrival, retention of reneged customers and finite buffer restriction. Bouchentouf and Guendouzi [4] offer a study where an $M^{X} / M / c$ Bernoulli feedback queuing system with variant multiple working vacations and impatience timers which depend on the states of the servers is considered. Using the probability generating functions (PGFs), they derive the steady-state solution of the model and then obtain useful performance measures. A numerical study is performed to explore the effects of the model parameters on the behaviour of the system along with an economic analysis. But their model neither assumes baulking, nor discourages arrival or retention of reneged customers, nor includes finite buffer restriction. Another relevant work is carried out by Bouchentouf, Yahiaoui, Kadi and Majid [5] where they consider the customers' impatience for a single server Markovian model under K-variant working vacation policy, waiting server, Bernoulli feedback, baulking, reneging, and retention of reneged customers. Using the probability-generating function (PGF) technique, they obtain the steady-state solution of the system. Useful performance measures of the considered queuing system are derived. A cost model was developed. The parameter optimisation is also carried out numerically, using a quadratic fit search method (QFSM). Finally, numerical examples are provided to visualise the analytical results. Even if the authors consider reneging, baulking, and retention of reneged customers, the concept of discouraged arrival is not applied. The reneging times are assumed to be exponentially distributed. They obtain the steadystate solution of the model and also derive some performance measures. The effect of the probability of retention on the average system size is studied. Some particular cases of the model are also derived and discussed. Awasthi [2] consider an $M / M / 1 / k$ queuing system with reverse baulking and reverse reneging. Steady-state solutions of 
the model are obtained and formulae for different performance measures are constructed. Fazlollahtabar and Gholizadeh [8] consider an $M / M / 1 / N$ queuing system with encouraged arrival. They describe the theory of queuing in a vague environment in which encourage arrivals rates and service rates are considered to be vague numbers. An economic analysis of the model is also presented by developing a cost model proceeding to exert uncertainty of the primary information when some of the parameters of the models are vague. A similar type of work is also carried out by Kordasiabi, Gholizadeh, Fazlollahtabar and Javadian [11] where they analyse the proficiency of a single server Markovian finite buffer queuing model with unpleasant services and encouraged arrival to compare the policies for service delivery. They also examine the economic analysis of the cost in an uncertain environment by considering arrival rate, service rate and the processing time as a vague number. Kumar, Som and Jain [13] considered an $M / M / 1 / N$ feedback queuing system with reverse baulking where they assume that feedback customer in queuing literature refers to a customer who is unsatisfied with incomplete, partial or unsatisfactory service and comes back to join the system. They obtain the steady-state solution and compute some performance measures of the model. But they do not consider the concept of reneging and discouraged arrival. A two-server Markovian queuing model with discouraged arrivals, reneging, and retention of reneged customers is analysed by Kumar and Sharma [14], but baulking is not assumed. In this paper, they consider a two heterogeneous server Markovian queuing model with discouraged arrivals, reneging, and retention of reneged customers. Here, they do not consider the other reflection of impatience, i.e., baulking and finite buffer restriction. The steady-state probabilities of system size are derived explicitly by using an iterative method. Some useful measures of effectiveness are also derived and discussed. Some queuing models are also derived as the special case of this model. Ammar and Sherbiny [1] study a single server finite capacity Markovian queue with discouraged arrivals and reneging, and obtain the transient solution of the model by matrix method. Kumar and Sharma $[12,15]$ consider the Markovian queueing system with discouraged arrivals and retention of reneged customers. Steady-state solutions are derived explicitly and cost-profit analysis of the model is also presented. Some measures of effectiveness are discussed. A special case of the model is also discussed. However, they did not include baulking, finite buffer restriction. Also, a closed-form expression of performance measures are not available. Rasheed and Manoharan [19] study a Markovian queueing system in which the arrival rate is state-dependent and whose service speed is regulated according to the number of customers in the system. Steady-state probabilities are derived and some performance measures such as expected number in the system/queue and expected waiting time in the system/queue are obtained. Multiple server discouraged arrival model having one service switch and single server discouraged arrival model having one and two services switches are obtained as special cases, but they did not assume customers' impatience. El-Paoumy and Nabwey [7] analyse $M / M / 2 / N$ queuing model with 
baulking, reneging, and heterogeneous server, and obtained the steady-state solution. But they do not consider discouraged arrival and retention of reneged customers.

From the review of the existing literature, it is clear that various queuing models have been analysed, considering customers' impatience. However, the concept of discouraged arrival and application of retention strategy finds little mention. Though a single server finite buffer Markovian model has been analysed, however, to the best of our knowledge, the said model under the assumption of discouraged arrival, retention of reneged customers, and state-dependent baulking have not been dealt with in the literature. Therefore, we try to address these gaps in this paper.

\section{The system-state probabilities}

In this section, the steady-state probabilities are derived by the Markov method. We first analyse the case where customers renege only from the queue. Under R_BOS, let $p_{n}$ denote the probability that there are $n$ customers in the system. The equations are:

$$
\begin{gathered}
\lambda p_{0}=\mu p_{1} \\
\lambda p_{0}+(\mu+v p) p_{2}=\frac{\lambda}{2}\left(1-\frac{1}{k}\right) p_{1}+\mu p_{1} \\
\frac{\lambda}{n}\left(1-\frac{n-1}{k}\right) p_{n-1}+(\mu+n v p) p_{n+1} \\
=\frac{\lambda}{n+1}\left(1-\frac{n}{k}\right) p_{n}+(\mu+(n-1) v p) p_{n}, \quad n=2, \ldots, k-1 \\
\frac{\lambda}{k}\left(1-\frac{k-1}{k}\right) p_{k-1}=(\mu+(k-1) v p) p_{k}, \quad n=k
\end{gathered}
$$

Solving recursively, we get (under R_BOS)

$$
p_{n}=\frac{\lambda^{n} \prod_{r=1}^{n}\left(1-\frac{r-1}{k}\right)}{\left.n ! \prod_{r=1}^{n}(\mu+(r-1) v p)\right)} \times p_{0}, \quad n=1,2, \ldots, k
$$


where $p_{0}$ is obtained from the normalising condition $\sum_{n=0}^{\infty} p_{n}=1$ and is given as

$$
p_{0}=\left[1+\sum_{n=1}^{k} \frac{\lambda^{n} \prod_{r=1}^{n}\left(1-\frac{r-1}{k}\right)}{n ! \prod_{r=1}^{n}\{\mu+(r-1) v p\}}\right]^{-1}
$$

Under R_EOS, where customers may renege from the queue as well as while being served, let $q_{n}$ denote the probability that there are $n$ customers in the system. Applying the Markov theory, we obtain the following set of steady-state equations:

$$
\begin{gathered}
\lambda q_{0}=(\mu+v p) q_{1} \\
\lambda q_{0}+(\mu+2 v p) q_{2}=\frac{\lambda}{2}\left(1-\frac{1}{k}\right) q_{1}+(\mu+v p) q_{1} \\
\frac{\lambda}{n}\left(1-\frac{n-1}{k}\right) q_{n-1}+(\mu+(n+1) \nu p) q_{n+1}=\frac{\lambda}{n+1}\left(1-\frac{n}{k}\right) q_{n} \\
+(\mu+n v p) q_{n}, n=2, \ldots, k-1 \\
\frac{\lambda}{k}\left(1-\frac{k-1}{k}\right) q_{k-1}=(\mu+k v p) q_{k}, n=k
\end{gathered}
$$

Solving recursively, we get (under R_EOS)

$$
q_{n}=\frac{\lambda^{n} \prod_{r=1}^{n}\left(1-\frac{r-1}{k}\right)}{n ! \prod_{r=1}^{n}(\mu+r v p)} \times q_{0}, \quad n=1,2, \ldots, k
$$

where $q_{0}$ is obtained from the normalizing condition $\sum_{n=0}^{\infty} q_{n}=1$ and is given as 


$$
q_{0}=\left[1+\sum_{n=1}^{k} \frac{\lambda^{n} \prod_{r=1}^{n}\left(1-\frac{r-1}{k}\right)}{n ! \prod_{r=1}^{n}(\mu+r v p)}\right]^{-1}
$$

\section{Performance measures}

Analysis of performance measures is important as these allow the cause of various queuing issues to be identified. Importantly, many of these are related to customers' dissatisfaction and are therefore of great interest to the management of the queuing system. We provide below the closed-form expression of some performance measures.

Mean system size $(\boldsymbol{L})$ and mean queue size $\left(\boldsymbol{L}_{\boldsymbol{q}}\right)$. An important measure in queuing system analysis is the mean number of customers in the system which is traditionally denoted by $L$. The derivation of mean system size $(L)$ and mean queue size $\left(L_{q}\right)$ for two reneging rules are presented in the appendix. Thus, mean system size and mean queue size under R_BOS are given by

$$
\begin{aligned}
& L_{\mathrm{R}_{-} \mathrm{BOS}}=\frac{1}{v p}\left(\frac{\lambda(k+1)}{k} \sum_{n=2}^{k} \frac{p_{n-1}}{n}+\frac{\lambda(k+1)}{k} p_{0}-(\mu-v p)\left(1-p_{0}\right)-\frac{\lambda}{k}\left(1-p_{k}\right)\right) \\
& L_{q\left(\mathrm{R} \_ \text {BOS }\right)}=\sum_{n=2}^{k}(n-1) p_{n}=L_{R_{-} B O S}+p_{0}-1 \\
&=\frac{1}{v p}\left(\frac{\lambda(k+1)}{k} \sum_{n=2}^{k} \frac{p_{n-1}}{n}+\frac{\lambda(k+1)}{k} p_{0}-\mu\left(1-p_{0}\right)-\frac{\lambda}{k}\left(1-p_{k}\right)\right)
\end{aligned}
$$

Under R_EOS, mean system size and mean queue size are given by

$$
L_{\mathrm{R} \_ \text {BOS }}=\frac{1}{v p}\left(\frac{\lambda(k+1)}{k} \sum_{n=2}^{k} \frac{q_{n-1}}{n}+\frac{\lambda(k+1)}{k} q_{0}-\mu\left(1-p_{0}\right)-\frac{\lambda}{k}\left(1-q_{k}\right)\right)
$$




$$
\begin{aligned}
L_{q\left(\mathrm{R} \_\mathrm{EOS}\right)}= & L_{\mathrm{R}_{-} \mathrm{EOS}}-1+q_{0}=\frac{1}{v p}\left(\frac{\lambda(k+1)}{k} \sum_{n=2}^{k} \frac{q_{n-1}}{n}+\frac{\lambda(k+1)}{k} q_{0}\right. \\
& \left.-(\mu+v p)\left(1-q_{0}\right)-\frac{\lambda}{k}\left(1-q_{k}\right)\right)
\end{aligned}
$$

Effective arrival rate $\left(\lambda^{e}\right)$. Customers who arrive in the system may not be able to join because of baulking and due to finite buffer restrictions. Moreover, we assume that customers arrive into the system at the rate $\lambda /(n+1)$. Thus, there exists a difference between the overall arrival rate and the effective arrival rate in the system. The closedform expression of the effective arrival rate under the two rules of reneging is given by

$$
\begin{aligned}
& \lambda_{\mathrm{R}_{\_} \text {вOS }}^{e}=\frac{\lambda(k+1)}{k} \sum_{n=2}^{k} \frac{p_{n-1}}{n}+\frac{(k+1) \lambda}{k} p_{0}-\frac{\lambda}{k}\left(1-p_{k}\right) \\
& \lambda_{\mathrm{R}_{-} \text {EOS }}^{e}=\frac{\lambda(k+1)}{k} \sum_{n=2}^{k} \frac{q_{n-1}}{n}+\frac{(k+1) \lambda}{k} q_{0}-\frac{\lambda}{k}\left(1-q_{k}\right)
\end{aligned}
$$

Average reneging rate (Avgrr). We assume that the customer who is at state $n$ has a random patience time following $\exp v$. Thus, the reneging rate of the system would depend on the state of the system as well as the reneging rule. The average reneging rate under both the reneging rule is given by

$$
\begin{aligned}
\operatorname{Avgrr~} \_ \text {BOS }_{-} & =\sum_{n=2}^{k}(n-1) v p p_{n}=v p L_{\mathrm{R} \_ \text {BOS }}-v p+v p p_{0} \\
& =\frac{\lambda(k+1)}{k} \sum_{n=2}^{k} \frac{p_{n-1}}{n}+\frac{(k+1) \lambda}{k} p_{0}-\frac{\lambda}{k}\left(1-p_{k}\right)-\mu\left(1-p_{0}\right) \\
{\operatorname{Avgrr~}{ }_{\text {R_EOS }}} & =\sum_{n=1}^{k} n v p q_{n}=v p L_{\mathrm{R} \_ \text {BOS }} \\
& =\frac{\lambda(k+1)}{k} \sum_{n=2}^{k} \frac{q_{n-1}}{n}+\frac{(k+1) \lambda}{k} q_{0}-\frac{\lambda}{k}\left(1-q_{k}\right)-\mu\left(1-q_{0}\right)
\end{aligned}
$$

Mean rate of losing a customer. In a real-life situation, customers who baulk or renege represent the business lost. Customers are lost to the system in three ways due to baulking, reneging and finite buffer restriction. Management would like to know the proportion of total customers lost to have an idea of total business lost. 
Hence the mean rate at which customers are lost (under R_BOS) is

$$
\lambda-\lambda_{\mathrm{R}_{-} \mathrm{BOS}}^{e}+\operatorname{Avgrr}_{\mathrm{R} \_ \text {BOS }}+A B R=\lambda-\mu\left(1-p_{0}\right)+\lambda \sum_{n=2}^{k} \frac{p_{n-1}}{k}
$$

and the mean rate at which customers are lost (under R_EOS) is

$$
\lambda-\lambda_{\mathrm{R}_{\_} \mathrm{BOS}}^{e}+\operatorname{Avgrr}_{\mathrm{R} \_ \text {BOS }}+A B R=\lambda-\mu\left(1-q_{0}\right)+\lambda \sum_{n=2}^{k} \frac{q_{n-1}}{k}
$$

where $\left(\lambda-\lambda^{e}\right)$ is loss due to finite buffer.

The proportion of customer lost and proportion of completing receipt of service can now be easily determined from the above results under R_BOS and R_EOS separately.

Actual load of the server. The customers who leave the system from the queue do not receive service. Consequently, only those customers who reach the service station constitute the actual load of the server. From the server's point of view, this provides a measure of the amount of work (s)he has to do. Let us call the rate at which customers reach the service station as $\lambda^{s}$. Then, under R_BOS ( 1 - proportion of customers lost due to reneging out of those joining the system) $\lambda_{\left(\mathrm{R}_{-} \mathrm{BOS}\right)}^{s}=\lambda_{\left(\mathrm{R}_{-} \mathrm{BOS}\right)}^{e}$

$$
\begin{aligned}
\lambda_{\mathrm{R}_{-} \mathrm{BOS}}^{s} & =\lambda_{\mathrm{R}_{-} \mathrm{BOS}}^{e}=\lambda_{\mathrm{R}_{-} \mathrm{BOS}}^{e}\left(\frac{1-\sum_{n=2}^{\infty}(n-1) v p p_{n}}{\lambda_{\mathrm{R}_{-} \mathrm{BOS}}^{e}}\right) \\
& =\lambda_{\mathrm{R}_{B} \mathrm{BOS}}^{e}-\operatorname{Avgrr}_{\mathrm{R}_{\_} \mathrm{BOS}}=\mu\left(1-p_{0}\right)
\end{aligned}
$$

In the case of R_EOS, one needs to recall that customers may renege even while being served and only those customers who renege from the queue will not constitute any work for the server. Thus, (1-proportion of customers lost due to reneging from the queue out of those joining the system)

$$
\begin{aligned}
\lambda_{\mathrm{R}_{\_} \text {EOS }}^{s}=\lambda_{\mathrm{R}_{\_} \text {EOS }}^{e} & =\lambda_{\mathrm{R}_{\_} \text {EOS }}^{e} \frac{1-\sum_{n=c+1}^{\infty}(n-1) v p q_{n}}{\lambda_{\text {(R_EOS })}^{e}} \\
& =\lambda_{\mathrm{R}_{-} \mathrm{EOS}}^{e}-v p\left(L_{\mathrm{R}_{-} \mathrm{EOS}}-q_{1}\right)+v p\left(1-q_{1}-q_{0}\right) \\
& =(\mu+v)\left(1-q_{0}\right)
\end{aligned}
$$


Average attrition rate $(\boldsymbol{A A R})$. Out of those customers who join the queuing system, some would leave after service and few others would leave because of impatience without service. Hence, management has to arrange sufficient infrastructural facilities to account for customers leaving the system through these two streams. We now present the expression for the average attrition rate $(A A R)$ under both the reneging rule.

$$
\begin{aligned}
A A R_{\mathrm{R}_{\_} \mathrm{BOS}}= & \mu p_{1}+(\mu+v p) p_{2}+\ldots+\{\mu+(k-1) v p\} p_{k} \lambda_{\mathrm{R}_{-} \mathrm{BOS}}^{s} \\
= & (\mu-v p)\left(1-p_{0}\right)+v p L_{\mathrm{R}_{-} \mathrm{BOS}} \\
A A R_{\mathrm{R}_{\_} \mathrm{EOS}} & =(\mu+v p) q_{1}+(\mu+2 v p) q_{2}+\ldots+\{\mu+k v p\} q_{k} \\
& =\mu\left(1-q_{0}\right)+v p L_{\mathrm{R}_{-} \mathrm{EOS}}
\end{aligned}
$$

Percentage reduction in attrition rate due to retention strategy out of those who had joined the system is given by

$$
\frac{A A R(p=1)-A A R(p<1)}{\operatorname{AAR}(p=1)}
$$

Average retention rate $(\boldsymbol{A R R})$. We recall that out of those customers who join the queuing system, some would renege and many others would be retained. It is therefore of interest to the management to have an idea of the rate at which customers are retained. We now present the expression for $A R R$ under R_BOS and R_EOS

$$
\begin{aligned}
& A R R_{\mathrm{R}_{\_} \mathrm{BOS}}=\sum_{n=2}^{k}(n-1) v q p_{n}= \frac{q}{p}\left(\frac{\lambda(k+1)}{k} \sum_{n=2}^{k} \frac{p_{n-1}}{n}+\frac{(k+1) \lambda}{k} p_{0}\right. \\
&\left.-\frac{\lambda}{k}\left(1-p_{k}\right)-v p p_{1}-(\mu-v q)\left(1-p_{0}\right)\right)-v q\left(1-p_{0}-p_{1}\right) \\
& A R R_{\mathrm{R}_{\_} \text {EOS }}=\sum_{n=2}^{k} n v q q_{n}= \frac{q}{p}\left(\frac{\lambda(k+1)}{k} \sum_{n=2}^{k} \frac{q_{n-1}}{n}+\frac{(k+1) \lambda}{k} q_{0}\right. \\
&\left.-\frac{\lambda}{k}\left(1-q_{k}\right)-v p p_{1}-\mu\left(1-p_{0}\right)\right)
\end{aligned}
$$

Proportion of customers retained due to retention strategy out of those who joined the system under both the reneging rule is given by 


$$
\frac{\operatorname{Avgrr}(p=1)-\operatorname{Avgrr}(p<1)}{\operatorname{Avgrr}(p=1)}
$$

Impact of discouraged arrival (DA). One of the features of our model is the assumption of discouraged arrival wherein the arrival rate of customers is a function of the state of the system. To give management a sense of business lost because of discouraged arrival we construct the following performance measure.

The proportion of customer lost due to discouraged arrival under both the reneging rule is given by

$$
\frac{\left.\lambda^{e} \text { (without } D A\right)-\lambda^{e}(\text { with } D A)}{\lambda^{e}(\text { without } D A)}
$$

\section{Numerical example}

To illustrate the use of our results, we apply them to a queuing scenario. We quote below an example from Taha [22].

The time for barber Joe to give a haircut is exponential with mean of 12 minutes. Because of his popularity, customers usually arrive (according to a Poisson distribution) at a rate much higher than Joe can handle 6 customers per hour. Joe really will feel comfortable if the arrival rate is effectively reduced to about 4 customers per hour. To accomplish this goal, he came up with the idea of providing limited seating in the waiting area so that newly arriving customers would go elsewhere when they discover that all the seats are taken. How many seats should Joe provide to accomplish his goal?

Solution. This is a design problem where the system manager (Joe, the barber) desires a system design in respect of the size of the waiting area (number of chairs for waiting customers).

Presently, $\lambda=6 / \mathrm{h}$ and $\mu=5 / \mathrm{h}$. As required by Joe, we examine the effect of limited seating arrangement in the waiting area with different choices of $k$. Though not explicitly stated, it is necessary to assume reneging and baulking. In today's competitive world, prompt customer service, being the expectation, is all the more reason to assume that customers are all of the reneging types. Since Joe has not collected data on customer reneging rate in his shop, we consider alternative possible Markovian reneging rates of $120 \min (v=0.5)$ and $100 \min (v=0.6)$. Also, the probability that a customer will renege is considered as $p=0.01$. Given the fact that service in a barbershop is being analysed, the reneging rule would be R_BOS. We further assume that the probability of baulking 
by an arriving customer is $i / k, i=1,2, \ldots, k$ where $i$ is the state of the customer observes the system to be in on its arrival.

Various performance measures of interest computed under different scenarios are given in Tables 1 and 2 . These measures were arrived at using a $\mathrm{C}++$ program coded by the authors. Different choices of $k$ were considered. Results relevant concerning Joe's desire to limit the arrival rate of customers into his service station to something around $4 / \mathrm{h}$ are presented in the tables.

Table 1. Performance measures assuming $\lambda=6 / \mathrm{h}, \mu=5 / \mathrm{h}, v=0.5 / \mathrm{h}$, and $p=0.01$

\begin{tabular}{|l|c|c|}
\hline \multirow{2}{*}{ Performance measure } & \multicolumn{2}{c|}{ Size of the waiting area } \\
\cline { 2 - 3 } & $\begin{array}{c}4 \\
k=5)\end{array}$ & $\begin{array}{c}5 \\
(k=6)\end{array}$ \\
\hline Arrival rate of customers reaching service station $\lambda^{s}$ & 3.98637 & 4.05979 \\
\hline Effective arrival rate $\lambda^{e}$ & 4.15665 & 4.66008 \\
\hline Fraction of time server is idle $p_{0}$ & 0.31618 & 0.295430 \\
\hline Average length of the queue & 1.02855 & 1.12922 \\
\hline Average length of the system & 1.46941 & 1.576872 \\
\hline Mean reneging rate & 0.52128 & 0.50529 \\
\hline Mean rate of customers lost & 1.94363 & 1.74021 \\
\hline $\begin{array}{l}\text { Proportion of customers lost due to reneging, } \\
\text { baulking, and finite buffer }\end{array}$ & 0.35179 & 0.33078 \\
\hline Average baulking rate & 0.45993 & 0.33999 \\
\hline Average retention rate & 0.78553 & 0.79958 \\
\hline Average attrition rate & 4.65666 & 4.75996 \\
\hline Proportion of customer lost due to discouraged arrival & 0.62609 & 0.63043 \\
\hline $\begin{array}{l}\text { Proportion of customer retained due to retention strategy } \\
\text { out of those who joined the system }\end{array}$ & 0.47597 & 0.56321 \\
\hline $\begin{array}{l}\text { Percentage reduction in attrition rate due to } \\
\text { retention strategy out of those who joined the system }\end{array}$ & 0.05462 & 0.06636 \\
\hline
\end{tabular}

Since a larger waiting area would also entail additional expenditure/investment, Joe needs to examine how the performance measures differ across different choices of $k$. In the case the reneging behaviour of customer follows exp0.5 distribution, it appears from Table 1 that an ideal choice of $k$ could be 5 (seating space in waiting area is 4 ) with $\lambda^{s}=3.98637$. In the case the reneging distribution is exp0.6, then $k=7$ appears to be close to Joe's target with $\lambda^{s}=4.00989$ (Table 2).

A few additional issues regarding the computed performance measures may be of interest:

- Numerically, the arriving rate of customers reaching the service station $\left(\lambda^{s}\right)$ has been computed to be lower than the effective arrival rate $\left(\lambda^{e}\right)$ both in Tables 1 and 2 . This is because some of the customers who join leave the system due to reneging and 
do not reach the service station. Thus, the number of customers who reach the service station are much less than those who join the system.

Table 2. Performance measures assuming $\lambda=6 / \mathrm{h}, \mu=5 / \mathrm{h}, v=0.6 / \mathrm{h}$, and $p=0.01$

\begin{tabular}{|l|c|c|}
\hline \multirow{2}{*}{\multicolumn{1}{|c|}{ Performance Measure }} & \multicolumn{2}{c|}{ Size of the waiting area } \\
\cline { 2 - 3 } & $\begin{array}{c}5 \\
k=6)\end{array}$ & $\begin{array}{c}6 \\
(k=7)\end{array}$ \\
\hline Arrival rate of customers reaching service station $\lambda^{s}$ & 3.45154 & 4.00989 \\
\hline Effective arrival rate $\lambda^{e}$ & 4.60658 & 4.69991 \\
\hline Fraction of time server is idle $p_{0}$ & 0.30616 & 0.29741 \\
\hline Average length of the queue & 1.02859 & 1.12923 \\
\hline Average length of the system & 1.56944 & 1.87691 \\
\hline Mean reneging rate & 0.57495 & 0.52973 \\
\hline Mean rate of customers lost & 1.96353 & 1.84012 \\
\hline $\begin{array}{l}\text { Proportion of customers lost due to reneging, } \\
\text { baulking, and finite buffer }\end{array}$ & 0.36389 & 0.34177 \\
\hline Average baulking rate & 0.41573 & 0.39673 \\
\hline Average retention rate & 0.79545 & 0.80695 \\
\hline Average attrition rate & 4.75656 & 4.85993 \\
\hline Proportion of customer lost due to discouraged arrival & 0.62771 & 0.63239 \\
\hline $\begin{array}{l}\text { Proportion of customer retained due to retention strategy } \\
\text { out of those who joined the system }\end{array}$ & 0.46988 & 0.55319 \\
\hline $\begin{array}{l}\text { Percentage reduction in attrition rate due to } \\
\text { retention strategy out of those who joined the system }\end{array}$ & 0.05893 & 0.06889 \\
\hline
\end{tabular}

- Joe aims to reduce his load from $6 / \mathrm{h}$ to $4 / \mathrm{h}$. In other words, he is not willing to serve $1 / 3$ rd of his potential customers. This aspect has been reflected in the proportion of customers lost due to reneging, baulking, and finite buffer.

- Because Joe serves fewer customers, he is idle about $30 \%$ of his time.

- The retention strategy adopted by Joe appears to be effective as the average retention rate is around $80 \%$.

- The impact of discouraged arrival appears to be substantial because more than $60 \%$ of the customers have been lost because of the same.

\section{Sensitivity analysis}

It is interesting to examine and understand how server utilisation varies in response to change in system parameters. The four system parameters of interest are $\lambda, \mu, v, k$. We place below the effect of change in these system parameters on server utilisation. For this purpose, we shall follow the following notational convention in the rest of this section. 
$p n(\lambda, \mu, v, k)$ and $q n(\lambda, \mu, v, k)$ will denote the probability that there are $n$ customers in a system with parameters $\lambda, \mu, v, k$ in steady state under R_BOS and R_EOS, respectively.

i) Let $\lambda_{1}>\lambda_{0}$, then

$$
\begin{aligned}
& \frac{p_{0}\left(\lambda_{1}, \mu, v, k\right)}{p_{0}\left(\lambda_{0}, \mu, v, k\right)}<1 \\
& \Rightarrow \frac{\left(\lambda_{0}-\lambda_{1}\right)}{\mu}+\frac{\left(\lambda_{0}{ }^{2}-\lambda_{1}{ }^{2}\right)\left(1-\frac{1}{k}\right)}{2 ! \mu(\mu+v p)}+\ldots+\frac{\left(\lambda_{0}{ }^{k}-\lambda_{1}{ }^{k}\right)\left(1-\frac{k-1}{k}\right)}{k ! \mu(\mu+v p) \ldots(\mu+(k-1) v p)}<0
\end{aligned}
$$

which is true. Hence, $p_{0}$ decreases as $\lambda$ increases.

ii) Let $\mu_{1}>\mu_{0}$, then

$$
\begin{aligned}
& \frac{p_{0}\left(\lambda, \mu_{1}, v, k\right)}{p_{0}\left(\lambda, \mu_{0}, v, k\right)}>1 \\
& \Rightarrow \lambda\left(\frac{1}{\mu_{0}}-\frac{1}{\mu_{1}}\right)+\frac{\lambda^{2}\left(1-\frac{1}{k}\right)}{2 !}\left(\frac{1}{\mu_{0}\left(\mu_{0}+v p\right)}-\frac{1}{\mu_{1}\left(\mu_{1}+v p\right)}\right)+\ldots \\
& +\frac{\lambda^{k}\left(1-\frac{k-1}{k}\right)}{k !}\left\{\frac{1}{\mu_{0}\left(\mu_{0}+v p\right) \ldots\left(\mu_{0}+(k-1) v p\right)}-\frac{1}{\mu_{1}\left(\mu_{1}+v p\right) \ldots\left(\mu_{1}+(k-1) v p\right)}\right\}+\ldots>0
\end{aligned}
$$

which is true. Hence, $p_{0}$ increases as $\mu$ decreases.

iii) Let $v_{1}>v_{0}$, then

$$
\begin{aligned}
& \frac{p_{0}(\lambda, \mu, v, k)}{p_{0}\left(\lambda, \mu, v_{0}, k\right)}>1 \Rightarrow \frac{\lambda^{2}\left(1-\frac{1}{k}\right)}{2 !}\left(\frac{1}{\mu\left(\mu+v_{0} p\right)}-\frac{1}{\mu\left(\mu+v_{1} p\right)}\right)+\ldots \\
& +\frac{\lambda^{k}\left(1-\frac{k-1}{k}\right)}{k !}\left(\frac{1}{\mu\left(\mu+v_{0} p\right) \ldots\left\{\mu+(k-1) v_{0} p\right\}}-\frac{1}{\mu\left(\mu+v_{1} p\right) \ldots\left\{\mu+(k-1) v_{1} p\right\}}\right)+\ldots>0
\end{aligned}
$$

which is true. Hence, $p_{0}$ increases as $v$ increases. 
iv) Let $k_{1}>k_{0}$, then

$$
\frac{p_{0}\left(\lambda, \mu, v, k_{1}\right)}{p_{0}\left(\lambda, \mu, v, k_{0}\right)}<1 \Rightarrow \sum_{n=1}^{k_{0}} \frac{\lambda^{n} \prod_{r=1}^{n}\left(1-\frac{r-1}{k}\right)}{n ! \prod_{r=1}^{n}(\mu+(r-1) v p)}-\sum_{n=1}^{k_{1}} \frac{\lambda^{n} \prod_{r=1}^{n}\left(1-\frac{r-1}{k}\right)}{n ! \prod_{r=1}^{n}(\mu+(r-1) v p)}<0
$$

which is true. Hence, $p_{0}$ decreases as $k$ increases.

Similar results are obtained under R_EOS. The managerial implications of the above results are obvious.

We can also study the variations in the other performance measures with response to change in system parameters numerically. We consider here the values of different system parameters from the numerical example mentioned in Section 5 under R_BOS. The results are computed using $\mathrm{C}++$ program.

Table 3. Variations in $L$, Avgrr, $p_{0}, A R R$, proportion of total lost, $\lambda^{s}$ and Effl with respect to mean arrival rate $\lambda$ considering $(\mu=5 / \mathrm{h}, v=0.5 / \mathrm{h}$, and $p=0.01, k=5)$

\begin{tabular}{|c|c|c|c|c|c|c|c|c|}
\hline$\lambda$ & $L$ & Avgrr & ARR & $p_{0}$ & AAR & $\begin{array}{c}\text { Proportion of } \\
\text { total lost }\end{array}$ & $\lambda^{s}$ & Effl \\
\hline 6 & 1.46941 & 0.52128 & 0.95453 & 0.31618 & 4.65666 & 0.35179 & 3.98637 & 4.15665 \\
\hline 7 & 1.87756 & 0.73041 & 1.53487 & 0.29993 & 4.82512 & 0.65296 & 4.24709 & 4.45126 \\
\hline 8 & 2.13671 & 0.90543 & 1.95786 & 0.26073 & 5.01871 & 0.808092 & 4.68172 & 4.97875 \\
\hline 9 & 2.45850 & 1.09067 & 2.42641 & 0.21738 & 5.35246 & 0.97065 & 4.91785 & 5.33327 \\
\hline 10 & 2.99862 & 1.3008 & 2.95634 & 0.17850 & 5.93860 & 1.14005 & 5.37797 & 5.938608 \\
\hline 11 & 3.77515 & 1.70111 & 3.56432 & 0.108562 & 6.20655 & 1.49582 & 5.81954 & 6.32065 \\
\hline
\end{tabular}

Table 4. Variations in $L$, Avgrr, $p_{0}, A R R$, proportion of total lost, $\lambda^{s}$ and Effl with respect to mean service rate $\mu$ considering $(\lambda=6, v=0.5, p=0.01, k=5)$

\begin{tabular}{|c|c|c|c|c|c|c|c|c|}
\hline$\mu$ & $L$ & Avgrr & ARR & $p_{0}$ & AAR & $\begin{array}{c}\text { Proportion of } \\
\text { total lost }\end{array}$ & $\lambda^{s}$ & Effl \\
\hline 5 & 1.46941 & 0.52128 & 0.95453 & 0.31618 & 4.65666 & 0.35179 & 3.98637 & 4.15665 \\
\hline 6 & 0.91633 & 0.48020 & 0.905638 & 0.46958 & 4.87588 & 0.30475 & 4.34738 & 4.64758 \\
\hline 7 & 0.60843 & 0.40015 & 0.734563 & 0.53307 & 5.31489 & 0.25717 & 4.79133 & 4.99149 \\
\hline 8 & 0.52855 & 0.36010 & 0.602341 & 0.58923 & 5.90363 & 0.20814 & 5.20362 & 5.40306 \\
\hline 9 & 0.46705 & 0.31009 & 0.516784 & 0.66502 & 6.13501 & 0.16253 & 5.69341 & 5.93501 \\
\hline 10 & 0.39831 & 0.26007 & 0.402387 & 0.77914 & 6.70342 & 0.11437 & 6.16695 & 6.29527 \\
\hline
\end{tabular}

From Table 3, it is clear that as the arrival rate increases, average system size, average reneging rate, $A R R, A A R$, the proportion of total customers lost due to baulking and reneging, rate at which customers reach the service system and effective arrival rate increase, which is quite obvious. The increase in average arrival rate means more customers in the system and it leads to a high level of impatience. Loss of customers means 
revenue loss for any system. Since the proportion of total customer lost increases with the increase in arrival rate so the system manager must appoint some additional server or have to increase the service rate to reduce the revenue loss.

From Table 4, it is evident that with the increase in an average rate of service, mean system size, average reneging rate, average baulking rate, $A R R$ and proportion of total customers lost due to impatience decreases. This means customers have to spend less time in the system and there will be fewer chances of getting impatient which is the most idealistic situation for any firm. On the other hand, the probability that the server is in idle condition, $A A R$ and effective arrival rate to the system increases with the increase in average service rate which is quite obvious. Therefore, we can say that the theoretical development in this paper is consistent with the proper functioning of the model.

Table 5. Variations in $L$, Avgrr, $p_{0}, A R R$, proportion of total lost, $\lambda^{s}$ and Effl with respect to the average reneging rate $v$ considering $(\lambda=6, \mu=5, p=0.01, k=5$

\begin{tabular}{|c|c|c|c|c|c|c|c|c|}
\hline$v$ & $L$ & Avgrr & ARR & $p_{0}$ & AAR & $\begin{array}{c}\text { Proportion of } \\
\text { total lost }\end{array}$ & $\lambda^{s}$ & Effl \\
\hline 0.5 & 1.46941 & 0.52128 & 0.95453 & 0.31618 & 4.65666 & 0.35179 & 3.98637 & 4.15665 \\
\hline 0.6 & 1.26882 & 0.69142 & 1.28472 & 0.37631 & 4.81935 & 0.40588 & 3.55576 & 4.47193 \\
\hline 0.7 & 0.86806 & 0.83084 & 1.78251 & 0.40648 & 5.15786 & 0.46041 & 2.95501 & 4.85786 \\
\hline 0.8 & 0.77325 & 1.38042 & 2.06743 & 0.43664 & 5.65852 & 0.50619 & 2.65426 & 5.35852 \\
\hline 0.9 & 0.66593 & 1.81668 & 2.69315 & 0.49680 & 5.95918 & 0.55635 & 2.25351 & 5.79186 \\
\hline 1.0 & 0.58867 & 2.23071 & 3.00683 & 0.51697 & 6.29845 & 0.59650 & 1.85277 & 6.05984 \\
\hline
\end{tabular}

Table 6. Variations in $L$, Avgrr, $p_{0}, A R R$, the proportion of total lost, $\lambda^{s}$ and Effl with respect to the system capacity $(k)$ considering $(\lambda=6, \mu=5, v=0.5, p=0.01)$

\begin{tabular}{|c|c|c|c|c|c|c|c|c|}
\hline$k$ & $L$ & Avgrr & ARR & $p_{0}$ & AAR & $\begin{array}{c}\text { Proportion of } \\
\text { total lost }\end{array}$ & $\lambda^{s}$ & Effl \\
\hline 5 & 1.46941 & 0.52128 & 0.95453 & 0.31618 & 4.65666 & 0.35179 & 3.98637 & 4.15665 \\
\hline 6 & 1.89734 & 0.73142 & 1.23481 & 0.30522 & 4.86512 & 0.39996 & 4.124701 & 4.51519 \\
\hline 7 & 2.63619 & 0.95825 & 1.67145 & 0.28073 & 5.03871 & 0.44807 & 4.618131 & 4.97871 \\
\hline 8 & 2.93841 & 1.15721 & 1.92641 & 0.21035 & 5.21269 & 0.49065 & 4.96924 & 5.23279 \\
\hline 9 & 3.33100 & 1.41023 & 2.35134 & 0.17361 & 5.75188 & 0.53955 & 5.247824 & 5.75560 \\
\hline 10 & 3.91595 & 1.93015 & 2.86492 & 0.11656 & 6.11671 & 0.61193 & 5.819543 & 6.23571 \\
\hline
\end{tabular}

It can be observed from Table 5 that the average reneging rate, the proportion of total customers lost and the probability that the system is in an empty state increase with the increase in average reneging rate. This is because as the average reneging rate of the system increases, more and more customers leave the queue without receiving service due to impatience. At the same time, this results in a decrease in average system size and the rate at which customers reach the server. 
From Table 6, it is clear that as the system capacity increases, average system size, average reneging rate, $A R R, A A R$, the proportion of total customer lost due to baulking and reneging, rate at which customers reach the service system and effective arrival rate also increase. If we increase the capacity of the system, that means we can accommodate more customers and it causes a high level of impatience among the customers. Loss of customers means the overall loss in the business. Since the proportion of total customer lost increases and the idle time for the server decreases with the increase in system capacity, so the system manager must not increase the system capacity or must appoint some additional server or have to increase the service rate to reduce the revenue loss.

\section{Conclusion}

The analysis of a single server finite buffer Markovian queueing system with discouraged arrival, state-dependent baulking, and retention of reneging has been presented. Closed-form expressions of several traditional, as well as novel performance measures, have been derived. To study the change in the system corresponding to change in system parameters, sensitivity analysis has also been presented. A numerical example has been discussed to demonstrate the obtained results. A numerical example of indicative nature is meant to illustrate the benefits of our theoretical results in a design context. The extension of our result considering general distribution is a pointer to future research.

\section{Appendix}

\section{A1. Derivation of $P^{\prime}(1)$ under R_BOS}

Let $\mathrm{P}(\mathrm{s})$ denote the probability generating function, defined by $P(s)=\sum_{n=0}^{\infty} p_{n} s^{n}$.

From equation (2), we have

$$
\lambda p_{0}+(\mu+v p) p_{2}=\frac{\lambda}{2}\left(1-\frac{1}{k}\right) p_{1}+\mu p_{1}
$$

Multiplying both sides of the equation by $s^{1}$ and summing over $n$

$$
\lambda s p_{0} s^{0}+\frac{1}{s}(\mu+v p) p_{2} s^{2}=\frac{\lambda}{2}\left(1-\frac{1}{k}\right) p_{1} s^{1}+\mu p_{1} s^{1}
$$


From (3) we have

$\frac{\lambda}{n}\left(1-\frac{n-1}{k}\right) p_{n-1}+(\mu+n v p) p_{n+1}=\frac{\lambda}{n+1}\left(1-\frac{n}{k}\right) p_{n}+(\mu+(n-1) v p) p_{n}, \quad n=2, \ldots, k-1$

Similarly, multiplying both sides of the equation by $s^{n}$ and summing over $n$

$$
\begin{gathered}
\sum_{n=2}^{k-1} \frac{\lambda\left(1-\frac{n-1}{k}\right)}{n} s p_{n-1} s^{n-1}-\sum_{n=2}^{k} \frac{\lambda\left(1-\frac{n}{k}\right)}{n+1} p_{n} s^{n}=\sum_{n=2}^{k-1}(\mu+(n-1) v p) p_{n} s^{n} \\
-\frac{1}{s} \sum_{n=2}^{k-1}(\mu+n v p) p_{n+1} s^{n+1}
\end{gathered}
$$

From (4) we have

$$
\frac{\lambda}{k}\left(1-\frac{k-1}{k}\right) p_{k-1}=(\mu+(k-1) v p) p_{k}
$$

Again, multiplying both sides of the equation by $s^{k}$

$$
\frac{\lambda}{k}\left(1-\frac{k-1}{k}\right) p_{k-1} s^{k-1}=(\mu+(k-1) \nu p) p_{k} s^{k}
$$

Adding (A1), (A2), and (A3)

$$
\begin{aligned}
\lambda s p_{0} s^{0} & +\lambda s \sum_{n=2}^{k-1}\left(1-\frac{n-1}{k}\right) \frac{p_{n-1} s^{n-1}}{n}+\frac{\lambda s\left(1-\frac{k-1}{k}\right)}{k} p_{k-1} s^{k-1} \\
& +\frac{1}{s}(\mu+v p) p_{2} s^{2}+\frac{1}{s} \sum_{n=2}^{k-1}(\mu+n v p) p_{n+1} s^{n+1}=\frac{\lambda\left(1-\frac{1}{k}\right)}{2} p_{1} s^{1} \\
& +\lambda \sum_{n=2}^{k-1}\left(1-\frac{n}{k}\right) \frac{p_{n} s^{n}}{n+1}+\mu p_{1} s^{1}+\sum_{n=2}^{k-1}\{\mu+(n-1) v p\} p_{n} s^{n}+\{\mu+(k-1) v p\} p_{k} s^{k}
\end{aligned}
$$




$$
\begin{aligned}
& \Rightarrow \lambda s p_{0} s^{0}+\lambda s \sum_{n=2}^{k} \frac{p_{n-1} s^{n-1}}{n}-\frac{\lambda s}{k}\left(\frac{1}{2} p_{1} s^{1}+\frac{2}{3} p_{2} s^{2}+\ldots+\frac{k-1}{k} p_{k-1} s^{k-1}\right) \\
& +\frac{\mu}{s}\left(P(s)-p_{1} s^{1}-p_{0} s^{0}\right)+\frac{v p}{s}\left(2 p_{2} s^{2}+\ldots+k p_{k} s^{k}-\left(p_{2} s^{2}+\ldots+p_{k} s^{k}\right)\right) \\
& =\lambda \sum_{n=2}^{k} \frac{p_{n-1} s^{n-1}}{n}-\frac{\lambda}{k}\left(\frac{1}{2} p_{1} s^{1}+\frac{2}{3} p_{2} s^{2}+\ldots+\frac{k-1}{k} p_{k-1} s^{k-1}\right) \\
& +\mu\left(P(s)-p_{0}\right)+v p\left(2 p_{2} s^{2}+\ldots+k p_{k} s^{k}-\left(p_{2} s^{2}+\ldots+p_{k} s^{k}\right)\right) \\
& \Rightarrow P^{\prime}(s) v p=\lambda \sum_{n=2}^{k} \frac{p_{n-1} s^{n-1}}{n}\left(1-\frac{1}{k}\right)-\frac{\lambda}{k}\left(P(s)-p_{k} s^{k}-p_{0}\right) \\
& -\frac{\mu}{s} P(s)+\frac{v p P(s)}{s}-\frac{v p p_{0}}{s}+\frac{\mu p_{0}}{s}
\end{aligned}
$$

Now,

$$
\begin{aligned}
\lim _{s \rightarrow-1} P^{\prime}(s)= & \lim _{\mathrm{s} \rightarrow-1} \frac{1}{v p}\left(\lambda \sum_{n=2}^{k} \frac{p_{n-1} s^{n-1}}{n}\left(1-\frac{1}{k}\right)-\frac{\lambda}{k}\left(P(s)-p_{k} s^{k}-p_{0}\right)\right. \\
& \left.-\frac{\mu}{s} P(s)+\frac{v p P(s)}{s}-\frac{v p p_{0}}{s}+\frac{\mu p_{0}}{s}\right) \\
\Rightarrow P^{\prime}(1)= & \frac{1}{v p}\left(\lambda \sum_{n=2}^{k} \frac{p_{n-1}}{n}\left(1+\frac{1}{k}\right)-\frac{\lambda}{k}\left(1-p_{k}-p_{0}\right)-\mu+\mu p_{0}+v p-v p p_{0}+\lambda p_{0}\right) \\
\Rightarrow P^{\prime}(1)= & \frac{1}{v p}\left(\lambda\left(\frac{k+1}{k}\right) \sum_{n=2}^{k} \frac{p_{n-1}}{n}+\frac{(k+1) \lambda}{k} p_{0}-\frac{\lambda}{k}\left(1-p_{k}\right)-(\mu-v p)\left(1-p_{0}\right)\right)
\end{aligned}
$$

\section{A2. Derivation of $Q^{\prime}(1)$ under R_EOS}

From equation (8) we have

$$
\lambda q_{0}+(\mu+2 v p) q_{2}=\frac{\lambda}{2}\left(1-\frac{1}{k}\right) q_{1}+(\mu+v p) q_{1}
$$


Multiplying both sides of the equation by $s^{1}$ and summing over $n$

$$
\lambda s q_{0} s^{0}+\frac{1}{s}(\mu+2 v p) q_{2} s^{2}=\frac{\lambda}{2}\left(1-\frac{1}{k}\right) q_{1} s^{1}+(\mu+v p) q_{1} s^{1}
$$

From (9) we have

$$
\frac{\lambda}{n}\left(1-\frac{n-1}{k}\right) q_{n-1}+(\mu+(n+1) v p) q_{n+1}=\frac{\lambda}{n+1}\left(1-\frac{n}{k}\right) q_{n}+(\mu+n v p) q_{n}, \quad n=2, \ldots, k-1
$$

Similarly, multiplying both sides of the equation by $s^{n}$ and summing over $n$

$$
\begin{aligned}
\sum_{n=2}^{k-1} \frac{\lambda\left(1-\frac{n-1}{k}\right)}{n} s q_{n-1} s^{n-1} & -\sum_{n=2}^{k} \frac{\lambda\left(1-\frac{n}{k}\right)}{n+1} q_{n} s^{n}=\sum_{n=2}^{k-1}(\mu+n v p) q_{n} s^{n} \\
& -\frac{1}{s} \sum_{n=2}^{k-1}(\mu+(n+1) v p) q_{n+1} s^{n+1}
\end{aligned}
$$

From (10) we have

$$
\frac{\lambda}{k}\left(1-\frac{k-1}{k}\right) q_{k-1}=(\mu+k v p) q_{k}
$$

Again, multiplying both sides of the equation by $s^{k}$

$$
\frac{\lambda}{k}\left(1-\frac{k-1}{k}\right) q_{k-1} s^{k-1}=(\mu+k v p) q_{k} s^{k}
$$

Adding (A4), (A5), and (A6) and proceeding like in Section (8.1), we obtain

$$
Q^{\prime}(1)=\frac{1}{v p}\left(\lambda\left(\frac{k+1}{k}\right) \sum_{n=2}^{k} \frac{p_{n-1}}{n}+\frac{(k+1) \lambda}{k} p_{0}-\frac{\lambda}{k}\left(1-p_{k}\right)-\mu\left(1-p_{0}\right)\right)
$$

\section{Acknowledgement}

The author likes to express his words of gratitude to Dr. Amit Choudhury, Department of Statistics, Gauhati University, India, for his guidance and support in carrying out this research work.

\section{References}

[1] Ammar S.I., El-Sherbiny A.A., Al-SeEdy R.O., A matrix approach for the transient solution of an M/M/1/N queue with discouraged arrivals and reneging, Int. J. Comp. Math., 2012, 89, 482-491, DOI: 10.1080/00207160.2011.637553. 
[2] AwASTHI B., Performance analysis of M/M/1/k finite capacity queuing model with reverse balking and reverse reneging, J. Comp. Math. Sci., 2018, 9 (7), 850-855.

[3] Bouchentouf A.A., MessabiHI A., Heterogeneous two server queuing system with reverse baulking and reneging, OPSERCH, 2018, 55, 251-267, DOI: 10.1007/s12597-017-0319-4.

[4] Bouchentouf A.A., Guendouzi A., The $M^{X} / M / c$ Bernoulli feedback queue with variant multiple working vacations and impatient customers: performance and economic analysis, Arab. J. Math., 2020, 9, 309-327, DOI: 10.1007/s40065-019-0260-x.

[5] Bouchentouf A.A., Yahiaoui L., Kadi M., Majid S., Impatient customers in Markovian queue with Bernoulli feedback and waiting server variant working vacation policy, Oper. Res. Dec., 2020, 30 (4), 6-28, DOI: 10.37190/ord200401.

[6] Bouchentouf A.A., YAHIAOUI L., On feedback queueing system with reneging and retention of reneged customers, multiple working vacations and Bernoulli schedule vacation interruption, Arab. J. Math., 2017, 6, 1-11, DOI: 10.1007/s40065-016-0161-1.

[7] El-Paoumy M.S., Nabwey H.A., The Poissonian queue with baulking function, reneging and two heterogeneous servers, Int. J. Basic Appl. Sci., 2011, 11 (6), 149-152.

[8] Fazlollahtabar H., Gholizadeh H., Economic analysis of the M/M/1/N queuing system cost model in a vague environment, Int. J. Fuzzy Logic Int. Syst., 2019, 19 (3), 192-203, DOI: 10.5391 /IJFIS.2019.19.3.192.

[9] HaIGHT F.A., Queuing with baulking, Biometrika, 1957, 44, 360-369, DOI: 10.1093/biomet/44.3-4.360.

[10] JAIN N.K., KUMAR R., SOM B.K., An M/M/1/N queuing system with reverse baulking, Am. J. Oper. Res., 2014, 2 (2), 17-20, https://www.ripublication.com/gjpam17/gjpamv13n7_49.pdf

[11] Kordasiabi M.C., Gholizadeh H., Fazlollahtabar H., Javadian N., Analysis of cost model with queuing system under uncertainty, J. Ind. Prod. Eng., 2020, DOI: 10.1080/21681015.2020.1797911.

[12] KumAR R., SHARMA S.K., A multi-server Markovian queuing system with discouraged arrivals and retention of reneged customers, Int. J. Oper. Res., 2012, 9 (4), 173-184.

[13] KumAr R., SOM B.K., JAIN S., An M/M/1/N feedback queuing system with reverse baulking, J. Rel. Stat. Stud., 2015, 8 (1), 31-38, http://www.jrss.in.net/assets/8104.pdf

[14] KUMAR R., SHARMA S.K., Two heterogeneous server Markovian queuing model with discouraged arrival, reneging and retention of reneged customers, Int. J. Oper. Res., 2014, 11 (2), 64-68, http:// orstw.org.tw/IJOR/vol11no2/IJOR2014_vol11_no2_p064_p068.pdf

[15] KUMAR R., SHARMA S.K., A single server Markovian queuing system with discouraged arrival and retention of reneged customers, Yugoslav J. Oper. Res., 2014, 1, 119-126, DOI: 10.2298/YJOR 120911019K.

[16] Medhi P., ChOudhury A., Aspects of impatience in a finite buffer queue, RAIRO Oper. Res., 2012, 46 (3), 189-209, DOI: 10.1051/ro/2012014.

[17] Medhi J., Stochastic Processes, 2nd Ed., New Age International (P), Ltd., 1994.

[18] NatVig B., On a queueing model where potential customers are discouraged by queue length, Scand. J. Stat., 1975, 2, 34-42.

[19] RASHEED K.V.A., MANOHARAN M., Markovian queueing system with discouraged arrivals and self-regulatory servers, Adv. Oper. Res., 2016, 1-11, DOI: 10.1155/2016/2456135.

[20] Saikia G., Medhi P., Choudhury A., Analyzing impatience in multiserver Markovian queues, Int. J. Suppl. Oper. Manage., 2020, 7, 310-321, DOI: 10.22034/IJSOM.2020.4.2.

[21] SOM B.K., KUMAR R., A heterogeneous queuing system with reverse baulking and reneging, J. Ind. Prod. Eng., 2017, 2 (2), 1-5, DOI: 10.1080/21681015.2017.1297739.

[22] TAHA H.A., Operations Research, 5th Ed., Prentice Hall of India Private Limited, 2003. 